\title{
Semigroup Actions on Intuitionistic Fuzzy Metric Spaces
}

\author{
Yaoyao Lan', ${ }^{1,}$ and Qingguo $\mathrm{Li}^{1}$ \\ ${ }^{1}$ Department of Mathematics and Economics, Hunan University, Changsha, Hunan 410082, China \\ ${ }^{2}$ Department of Mathematics and Statistics, Chongqing University of Arts and Sciences, Yongchuan, \\ Chongqing 402160, China
}

Correspondence should be addressed to Yaoyao Lan, lyygut@yahoo.com.cn

Received 25 March 2009; Accepted 19 May 2009

Recommended by Zne-Jung Lee

This paper investigates the dynamical systems in the context of topological semigroup actions on intuitionistic fuzzy metric spaces. We give some concepts such as topological transitivity, point transitivity, and densely point transitivity for such dynamical systems. Particularly, we consider the implications of nonsensitivity and its relation to dynamical properties such as transitivity and equicontinuity.

Copyright (C) 2009 Y. Lan and Q. Li. This is an open access article distributed under the Creative Commons Attribution License, which permits unrestricted use, distribution, and reproduction in any medium, provided the original work is properly cited.

\section{Introduction}

As a generalization of fuzzy sets [1], the concepts of intuitionistic fuzzy sets was introduced by Atanassov [2], and later there has been much progress in the study of intuitionistic fuzzy sets by many authors [3-6]. Following George and Veeramani [7], Park [8] has defined intuitionistic fuzzy metric spaces and obtained several classical theorems on this new structure. Furthermore, Alaca et al. [9] studied new properties of intuitionistic fuzzy metric spaces. This concept has very important applications in quantum particle physics particularly in connection with both string and $\epsilon^{\infty}$ theory which were given and studied by El Naschie $[10,11]$ and by Sigalotti and Mejias [12].

On the other hand, the traditional theory of dynamical systems is founded on topology and gives topological descriptions of orbits, recurrence and stability. However, even the probabilistic approach cannot represent uncertainties attached to systems where some deterministic dynamical characteristics are unknown or deliberately ignored as well as uncertainties attached to their mathematical model. This observation led Chang and Zadeh to the concepts of fuzzy systems [13]. Many efforts have been made in this direction [14-16].

Recently, an enormous amout of work has been conducted on chaotic dynamical systems. Most of this work has been concerned with the iteration of single maps, in other words, with group (or semigroup) actions of the additive group [17-20]. However, the abovementioned work has been studied mainly in the context of classical metric spaces.

In this paper, we consider the dynamical systems in the context of topological semigroup actions on intuitionistic fuzzy metric spaces. We discuss topological transitivity, point transitivity, densely point transitivity and equicontinuity for such dynamical systems. We also investigate the relations between nonsensitivity and some dynamical properties such as transitivity and equicontinuity.

\section{Preliminaries}

Definition 1. A binary operation $*:[0,1] \times[0,1] \rightarrow[0,1]$ is continuous $t$-norm if $*$ satisfies the following conditions:

(a) $*$ is commutative and associative,

(b) $*$ is continuous,

(c) $a * 1=a$ for all $a \in[0,1]$,

(d) $a * b \leq c * d$ whenever $a \leq c$ and $b \leq d$, for each $a, b, c, d \in[0,1]$.

Definition 2. A binary operation $\diamond:[0,1] \times[0,1] \rightarrow[0,1]$ is continuous $t$-conorm if $\diamond$ satisfies the following conditions: 
(a) $\diamond$ is commutative and associative,

(b) $\diamond$ is continuous,

(c) $a \diamond 0=a$ for all $a \in[0,1]$

(d) $a \diamond b \leq c \diamond d$ whenever $a \leq c$ and $b \leq d$, for each $a, b, c, d \in[0,1]$.

Definition 3. A 5-tuple $(X, M, N, *, \diamond)$ is called intuitionistic fuzzy metric space if $X$ is an arbitrary set, $*$ is a continuous $t$-norm, $\diamond$ is a continuous $t$-conorm and $M, N$ are fuzzy sets on $X^{2} \times(0, \infty)$ satisfying the following conditions: for all $x, y, z \in X, s, t>0$,

$$
\begin{aligned}
& -(\text { IFm-1): } M(x, y, t)+N(x, y, t) \leq 1, \\
& -(\text { IFm-2): } M(x, y, t)>0, \\
& \text { - (IFm-3): } M(x, y, t)=1 \text { if and only if } x=y, \\
& -(\text { IFm-4): } M(x, y, t)=M(y, x, t), \\
& -(\text { IFm-5): } M(x, y, t) * M(y, z, s) \leq M(x, z, t+s), \\
& \text { - (IFm-6): } M(x, y, t):(0, \infty) \rightarrow(0,1] \text { is continuous, } \\
& \text { - (IFm-7): } N(x, y, t)<1, \\
& \text { - (IFm-8): } N(x, y, t)=0 \text { if and only if } x=y, \\
& -(\text { IFm-9): } N(x, y, t)=N(y, x, t), \\
& -(\text { IFm-10): } N(x, y, t) \diamond N(y, z, s) \geq N(x, z, t+s), \\
& -(\text { IFm-11): } N(x, y, t):(0, \infty) \rightarrow(0,1] \text { is continuous. }
\end{aligned}
$$

Then $(M, N)$ is called an intuitionistic fuzzy metric on $X$. The function $M(x, y, t)$ and $N(x, y, t)$ denote the degree of nearness and the degree of nonnearness between $x$ and $y$ respect to $t$, respectively.

Remark 1. In intuitionistic fuzzy metric spaces $X, M(x, y, \cdot)$ is nondecreasing and $N(x, y, \cdot)$ is nonincreasing for all $x, y \in$ $X$.

\section{Main Results}

For brevity, we write the intuitionistic fuzzy metric spaces as $X$, wherever there is no risk of confusion.

Definition 4. A dynamical system in $X$ is a triple $(S, X, \pi)$ (we note $(S, X)$ for short), where $S$ is a topological semigroup, $X$ at least is Hausdorff and

$$
\begin{gathered}
\pi: S \times X \longrightarrow X \\
(s, x) \mapsto s x
\end{gathered}
$$

is a continuous action on $X$. Thus, $s_{1}\left(s_{2} x\right)=\left(s_{1} s_{2}\right) x$ holds for each $\left(s_{1}, s_{2}, x\right)$ in $S \times S \times X$. The orbit of $x$ is the set $S x=\{s x$ : $s \in S\}$. A subset $Y \subset X$ is said to be $S$-invariant if $f(Y) \subset Y$. If $Y$ is a closed $S$-invariant subset of $X$, then we call $(S, Y)$ the restricted action, is a subsystem of $(S, X)$. For $U \subset X$ and $s \in S$ denote $s^{-1} U=\{x \in X: s x \in U\}$.

Note that if $S=\left\{f^{n}: n=1,2, \ldots\right\}$ and $f: X \rightarrow X$ is continuous, then $(S, X)$ is a classical dynamical system in $X$. Notation: $(X, f)$.
Definition 5. Let $S$ be a topological semigroup, we say that $S$ is an

(1) F-semigroup if for every $s_{0} \in S$ the subset $S \backslash S s_{0}$ is finite,

(2) $C$-semigroup if for every $s_{0} \in S$, the closure of the subset $S \backslash S s_{0}$ is compact in $S$ (i.e., $S \backslash S s_{0}$ is relatively compact).

Obviously, every topological group is of course an $\mathrm{F}$ semigroup and also a $C$-semigroup.

Definition 6. Let $(S, X)$ be a dynamical system.

(1) A subset $A$ of $S$ acts equicontinuously at $x_{0} \in X$ if for every $\varepsilon \in(0,1)$ and $t>0$, there exists $\delta \in(0,1)$ such that $M\left(x_{0}, x, t\right)>\delta$ and $N\left(x_{0}, x, t\right)<1-\delta$ imply $M\left(a x_{0}, a x, t\right)>\varepsilon$ and $N\left(x_{0}, x, t\right)<1-\varepsilon$ for every $a \in A$.

(2) A point $x_{0} \in X$ is a point of equicontinuity (notation: $\left.x_{0} \in \mathrm{Eq}(X)\right)$ if $S$ acts equicontinuously at $x_{0} .(S, X)$ is equicontinuous if $\mathrm{Eq}(X)=X$.

(3) $(S, X)$ is almost equicontinuous if $\mathrm{Eq}(X)$ is dense in $X$.

Observe that every equicontinuous system is almost equicontinuous. Theorem 3 show that the relation between almost equicontinuity and nonsensitivity.

Lemma 1. Let $(S, X)$ be a dynamical system. If $A$ is a related compact subset of $S$, then $A$ acts equicontinuously on $X$.

Proof. Let $x \in X$ and $s_{1} \in S$. Suppose that $s_{2} \in S$ is in the neighborhood of $s_{1}$ defined by $M\left(s_{1} x, s_{2} x, t\right)>r$ and $N\left(s_{1} x, s_{2} x, t\right)<1-r$ for some $r \in(0,1)$ and $t>0$. Given $\varepsilon>0$. Note that $\pi(s, x)=s x$, by the continuity of $\pi$, for each $s \in S$ there is an open neighborhood $U_{s}$ of $s$ and a $\delta_{s}>0$ such that if $s^{\prime} \in U_{s}$ and $y \in B\left(x, \delta_{s}, t\right)$, then

$$
\begin{array}{ll}
M\left(s^{\prime} y, s x, t\right)>\varepsilon, & N\left(s^{\prime} y, s x, t\right)<1-\varepsilon, \\
M\left(s x, s^{\prime} x, t\right)>\varepsilon, & N\left(s x, s^{\prime} x, t\right)<1-\varepsilon
\end{array}
$$

Therefore,

$$
\begin{aligned}
M\left(s^{\prime} x, s^{\prime} y, 2 t\right) & \geq M\left(s^{\prime} y, s x, t\right) * M\left(s x, s^{\prime} x, t\right) \\
& >\varepsilon * \varepsilon>\varepsilon_{1} \quad \text { for some } 0<\varepsilon_{1}<1, \\
N\left(s^{\prime} x, s^{\prime} y, 2 t\right) & \leq N\left(s^{\prime} y, s x, t\right) \diamond N\left(s x, s^{\prime} x, t\right) \\
& <(1-\varepsilon) \diamond(1-\varepsilon) \\
& <1-\varepsilon_{2} \quad \text { for some } 0<\varepsilon_{2}<1 .
\end{aligned}
$$

Taking $\varepsilon^{\prime}=\max \left\{\varepsilon_{1}, \varepsilon_{2}\right\}$ and $t^{\prime}=2 t$, thus we have $M\left(s^{\prime} x\right.$, $\left.s^{\prime} y, t^{\prime}\right)>\varepsilon^{\prime}$ and $N\left(s^{\prime} x, s^{\prime} y, t^{\prime}\right)<1-\varepsilon^{\prime}$. From the compactness of $\bar{A}$ it easily follows that there is a $\delta>0$ such that if $s^{\prime} \in S$, $M(x, y, t)>\delta$ and $N(x, y, t)<1-\delta$ then

$$
M\left(s^{\prime} x, s^{\prime} y, t^{\prime}\right)>\varepsilon^{\prime}, \quad N\left(s^{\prime} x, s^{\prime} y, t^{\prime}\right)<1-\varepsilon^{\prime} .
$$

Hence $x \in \mathrm{Eq}(X)$. 
Definition 7. The dynamical system $(S, X)$ is called as follows.

(1) TT: topological transitive if for every pair of nonempty subsets $U$ and $V$ in $X$, there exists $s \in S$ such that $s U \cap V \neq \varnothing$.

(2) PT: point transitivity if $S$ has a dense orbit, that is, there is a point $x_{0} \in X$ whose orbit is dense in $X$. Such a point is called transitive point. Notation: $x_{0} \in$ $\operatorname{Trans}(X)$.

(3) DPT: densely point transitive if there is a dense set of transitive point, that is, $\operatorname{Trans}(X)$ is dense in $X$.

Remark 2. (1) One can easily verify that $s\left(s^{-1} V \cap U\right)=V \cap$ $s U \neq \varnothing$, it is say that $U \cap s^{-1} V \neq \varnothing$.

(2) Clearly, DPT implies PT. Generally, TT and PT are independent properties.

Proposition 1. Let $(S, X)$ be a dynamical system.

(1) Let $X$ be perfect (has no isolated point) and $S$ an Fsemigroup. Then PT implies TT. Furthermore, if $X$ is separable and second category, then TT implies DPT and hence also PT.

(2) Every DPT system $(S, X)$ is TT.

Proof. (1) Let $x$ be a transitive point with dense orbit $S x$ and $(U, V)$ a pair of nonempty open subset of $X$, then there exists $s_{1} \in S$ such that $s_{1} x \in U$. Since $S$ is an $F$-semigroup, the subset $S \backslash S s_{1}$ is finite. On the other hand, $S s_{1} x$ is dense in $X$. Indeed, removing the finite subset $\left(S \backslash S s_{1}\right) x$ from the dense orbit $S x$ we obtain again a dense subset because $X$ has no isolated point. Then there exists $s_{2} \in S$ such that $s_{2} s_{1} x \in$ $V$, that is, $s_{1} x \in s_{2}^{-1} V$. Thus, $s_{1} x \in U \cap s_{2}^{-1} V$ and hence $U \cap s_{2}^{-1} V \neq \varnothing$.

For the second half, assume that $S$ has no dense orbit and $\left\{U_{n}\right\}_{n=1}^{\infty}$ is a countable base. For each $x \in X$ there is some $U_{n}$ such that $s x \notin U_{n}$. But $\bigcup_{s \in S} s U$ is open and meets every open set in $X$ since $(S, X)$ is $T T$. Let $V_{n}=X \backslash \bigcup_{s \in S} s U$, then $x \in V_{n}$ and $V_{n}$ is closed and has empty interior. However, $X=\bigcup V_{n}$ is a countable union, contradicting the fact that $X$ is of second category.

(2) Let $U$ and $V$ be nonempty open subsets of $X$. Since $(S, X)$ is $D P T$, we can choose a transitive point $x_{0} \in U$. On the other hand, there exists $s \in S$ such that $s x_{0} \in V$ since the orbit of $x_{0}$ is dense in $X$. Therefore, $s x_{0} \in s U \cap V$.

Proposition 2. If the dynamical system $(S, X)$ is $T T$, then $E q(X) \subset \operatorname{Trans}(X)$.

Proof. Let $x_{0} \in \mathrm{Eq}(X)$ and $y \in X$. For the orbit $S x_{0}$ of $x_{0}$ and the $r$-neighborhood $B(y, r, t)=\{z \in X: M(y, z, t)>$ $r, N(y, z, t)<1-r\}$ of $y$, we show that $s x_{0} \cap B(y, r, t) \neq \varnothing$ for $r \in(0,1)$ and $t>0$. Since $x_{0} \in \mathrm{Eq}(X)$, there exists a neighborhood $U$ of $x_{0}$ such that

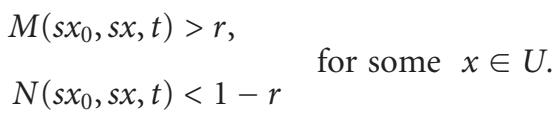

Therefore,

$$
\begin{aligned}
M\left(s_{0} x_{0}, y, 2 t\right) & \geq M\left(s_{0} x_{0}, s x, t\right) * M\left(s_{0} x, y, t\right) \\
& >r * r>r_{1} \quad \text { for some } 0<r_{1}<1, \\
N\left(s_{0} x_{0}, y, 2 t\right) & \leq N\left(s_{0} x_{0}, s x, t\right) \diamond N\left(s_{0} x, y, t\right) \\
& <(1-r) \diamond(1-r) \\
& <r_{2} \quad \text { for some } 0<r_{2}<1 .
\end{aligned}
$$

Taking $r^{\prime}=\max \left\{r_{1}, r_{2}\right\}$ and $t^{\prime}=2 t$, we then have $M\left(s_{0} x_{0}, y, t^{\prime}\right) \geq r^{\prime}$ and $N\left(s_{0} x_{0}, y, t^{\prime}\right) \leq 1-r^{\prime}$. This completes the proof.

Proposition 3. Let $S$ be a C-semigroup. If $(S, X)$ is $P T$ and $E q(X) \neq \varnothing$, then $\operatorname{Trans}(X) \subset E q(X)$, that is, every transitive point is an equicontinuous point.

Proof. Let $y \in \operatorname{Trans}(X)$ and $x \in \operatorname{Eq}(X)$. We claim that $y \in$ $\mathrm{Eq}(X)$. For given $r>0$ there is a neighborhood $O(x)$ of $x$ such that

$$
\begin{aligned}
& M\left(s x^{\prime \prime}, s x^{\prime}, t\right)>\varepsilon, \\
& N\left(s x^{\prime \prime}, s x^{\prime}, t\right)<1-\varepsilon
\end{aligned}
$$

for all $\left(s, x^{\prime}, x^{\prime \prime}\right) \in S \times O(x) \times O(x)$. Since $y \in \operatorname{Trans}(X)$ so there exists $s_{0} \in S$ such that $s_{0} y \in O(x)$. Thus $O(y)=s_{0}^{-1} O(x)$ is a neighborhood of $y$. Therefore, for each $\left(s, y^{\prime}, y^{\prime \prime}\right) \in S \times O(y) \times O(y)$,

$$
M\left(s s_{0} y^{\prime}, s s_{0} y^{\prime \prime}, t\right)>\varepsilon, \quad N\left(s s_{0} y^{\prime}, s s_{0} y^{\prime \prime}, t\right)<1-\varepsilon .
$$

Since $S$ is a $C$-semigroup the subset $M=\overline{S \backslash S s_{0}}$ is compact. By Lemma $1, S$ acts equicontinuously on $(S, X)$. Hence we can choose a neighborhood $U_{y}$ of $y$ such that for all $\left(m, y^{\prime}, y^{\prime \prime}\right) \in M \times U(y) \times U(y)$,

$$
M\left(m y^{\prime}, m y^{\prime \prime}, t\right)>\varepsilon, \quad N\left(m y^{\prime}, m y^{\prime \prime}, t\right)<1-\varepsilon .
$$

Let $V=O(y) \cap U(y)$, obviously $V$ is a neighborhood of $y$. Since $S=S s_{0} \cup M$, we have for each $\left(s, y^{\prime}, y^{\prime \prime}\right) \in S \times V \times V$,

$$
M\left(s y^{\prime}, s y^{\prime \prime}, t\right)>\varepsilon, \quad N\left(s y^{\prime}, s y^{\prime \prime}, t\right)<1-\varepsilon .
$$

This proves that $y \in \operatorname{Eq}(X)$ and hence $\operatorname{Trans}(X) \subset \operatorname{Eq}(X)$.

Definition 8. A (not necessarily compact) dynamical system $(S, X)$ is called minimal if every point of $X$ is transitive.

Theorem 1. Let $S$ be a $C$-semigroup. If $(S, X)$ is minimal and $E q(X) \neq \varnothing$, then $X$ is equicontinuous.

Proof. By the definition of minimal, we have $\operatorname{Trans}(X)=X$. Using Proposition 3, if $\mathrm{Eq}(X) \neq \varnothing$ then every transitive point is an equicontinuity point. Thus, $\mathrm{Eq}(X)=X$.

Theorem 2. Let $S$ be a C-semigroup. Assume that the dynamical system $(S, X)$ is separable and second category. Then $(S, X)$ is almost equicontinuous if and only if $\mathrm{E} q(X) \neq \varnothing$. 
Proof. Clearly, if $(S, X)$ is almost equicontinuous then $\operatorname{Eq}(X) \neq \varnothing$. On the other hand, using Proposition 1(1), we obtain that $(S, X)$ is $D P T$, that is, $\operatorname{Trans}(X)$ is dense in $X$. If $\operatorname{Eq}(X) \neq \varnothing$, then $\operatorname{Trans}(X) \subset \mathrm{Eq}(X)$ by Proposition 3, it follows that $\operatorname{Eq}(X)$ is also dense in $X$ and hence $(S, X)$ is almost equicontinuous.

Definition 9 (sensitive dependence on initial conditions). A dynamical system $(S, X)$ has sensitive dependence on initial conditions or more briefly, is sensitive, if there exists $\varepsilon \in$ $(0,1)$ and $t>0$ such that for every $x \in X$ and every neighborhood $U$ of $x$, there exists $(s, y) \in S \times U$ with

$$
M(s x, s y, t)<\varepsilon, \quad N(s x, s y, t)>1-\varepsilon .
$$

When $(S, X)$ is not sensitive, we say that $(S, X)$ is nonsensitive.

Remark 3. (1) The definition of sensitive dependence on initial conditions plays an important role in classical chaotic systems. Note that the above form is just a generalization of existing definition for $(X, f)$ when $S=\left\{f^{n}: n=1,2 \ldots\right\}$.

(2) Spelling the property nonsensitive out we have: for every $\varepsilon \in(0,1)$ and $t>0$, there exists $x \in X$ and a neighborhood $U$ of $x$, such that for each $(s, y) \in S \times U$,

$$
M(s x, s y, t) \geq \varepsilon, \quad N(s x, s y, t) \leq 1-\varepsilon .
$$

We observe that trivially $(S, X)$ is nonsensitive whenever $X$ has an isolated point.

(3) Without loss of generality, we sometimes use the open ball $B(x, r, t)$ instead of the neighborhood $U$ of $x$ in Definition 9.

Proposition 4. For a PT dynamical system $(S, X)$ with no isolated points, being nonsensitive is equivalent to the following property: for every $\varepsilon \in(0,1)$ and $t>0$, there exists a transitive point $x_{0} \in X$ and a neighborhood $U$ of $x_{0}$ such that for every $y \in U$ and every $s \in S$,

$$
M\left(s x_{0}, s y, t\right) \geq \varepsilon, \quad N\left(s x_{0}, s y, t\right) \leq 1-\varepsilon .
$$

Proof. Let $\varepsilon^{\prime}$ be given and let $x$ and $U$ be as in the definition of nonsensitive. Since $(S, X)$ is $P T$, there is a point $x_{0}$ in $X$ whose orbit is dense, that is, there exists a $s_{0} \in S$ such that $s_{0} x_{0} \in U$. Denote $x_{1}=s_{0} x_{0}$, then

$$
M\left(s x, s x_{1}, t\right) \geq \varepsilon^{\prime}, \quad N\left(s x, s x_{1}, t\right) \leq 1-\varepsilon^{\prime} .
$$

On the other hand, there exists $r \in(0,1)$ and $t>0$ such that $B(x, r, t) \subset U$. Let $V=B(x, r, t)$, then for every $y \in V \subset U$ and $s \in S$,

$$
M(s x, s y, t) \geq \varepsilon^{\prime}, \quad N(s x, s y, t) \leq 1-\varepsilon^{\prime} .
$$

Therefore,

$$
M\left(s x_{1}, s y, 2 t\right) \geq M\left(s x, s x_{1}, t\right) * M(s x, s y, t) \geq \varepsilon^{\prime} * \varepsilon^{\prime} \geq \varepsilon_{1}
$$

for some $\varepsilon_{1} \in(0,1)$, and

$$
\begin{aligned}
N\left(s x_{1}, s y, 2 t\right) & \leq N\left(s x, s x_{1}, t\right) \diamond N(s x, s y, t) \\
& \leq\left(1-\varepsilon^{\prime}\right) \diamond\left(1-\varepsilon^{\prime}\right) \leq 1-\varepsilon_{2}
\end{aligned}
$$

for some $\varepsilon_{2} \in(0,1)$.
Taking $\varepsilon=\max \left\{\varepsilon_{1}, \varepsilon_{2}\right\}$ and $t^{\prime}=2 t$, then $M\left(s x_{1}, s y, t^{\prime}\right) \geq$ $\varepsilon$ and $N\left(s x_{1}, s y, t^{\prime}\right) \leq 1-\varepsilon$. Since $X$ has no isolated points, the point $x_{1}$ is also transitive and the proof is complete.

Theorem 3. Let $S$ be a C-semigroup. Assumed that a TT dynamical system $(S, X)$ is separable and second category. Then $(S, X)$ is almost equicontinuous if and only if it is nonsensitive.

Proof. Suppose that $\left\{U_{n}\right\}_{n=1}^{\infty}$ is a countable base. Since $(S, X)$ is nonsensitive, for any $\varepsilon \in(0,1)$, there exists an $x \in X$ and a neighborhood $U$ of $x$ such that for all $(s, y) \in S \times U$ and $t>0$,

$$
M(s x, s y, t) \geq \varepsilon, \quad N(s x, s y, t) \leq 1-\varepsilon .
$$

Since $\left\{U_{n}\right\}_{n=1}^{\infty}$ is a base, there exists $n_{0} \in \mathbb{N}$ such that $y \in$ $U_{n_{0}} \subset U$. Without loss of generality, we can assume that $s U_{n} \subset B(s x, 1 / n, t)$, for all $(s, n) \in S \times \mathbb{N}$. Let

$$
V_{n}=S^{-1} U_{n}, \quad M=\bigcap_{n \in \mathbb{N}} V_{n}
$$

Clearly, every $V_{n}$ is open and meets every open subset of $X$ since $(S, X)$ is TT. This means that each $V_{n}$ is dense in $X$. Since $X$ is second category, by Baire theorem, $M=\bigcap_{n \in \mathbb{N}} V_{n}$ is also dense. Now it remains to show that $M \subset \mathrm{Eq}(X)$. Given $x \in M$, then there exists $s_{0} \in S$ such that $s_{0} x \in U_{n}$. Let $V=s_{0}^{-1} U_{n}$. Hence for $y \in V$ and each $s=s^{\prime} s_{0} \in S s_{0}$ we have

$$
M(s x, s y, t)=M\left(s^{\prime} s_{0} x, s^{\prime} s_{0} y, t\right) \geq \frac{1}{n} \geq r_{1}
$$

for some $r_{1} \in(0,1)$ and

$$
N(s x, s y, t)=N\left(s^{\prime} s_{0} x, s^{\prime} s_{0} y, t\right) \leq 1-\frac{1}{n} \leq r_{2}
$$

for some $r_{2} \in(0,1)$.

Taking $r=\max \left\{r_{1}, r_{2}\right\}$ then $M(s x, s y, t) \geq r$ and $N(s x, s y, t) \leq 1-r$. But $\overline{S \backslash S s_{0}}$ is compact because $S$ is an $C$-semigroup. Then by Lemma 1 , the set $\overline{S \backslash S s_{0}}$ acts equicontinuously on $(S, X)$. This means that if $W$ is an open neighborhood of $x$, then for all $y \in W$ and for each $s \in$ $\overline{S \backslash S s_{0}}$,

$$
M(s x, s y, t) \geq r, \quad N(s x, s y, t) \leq 1-r
$$

holds. Let $O=W \cap V$ be an open neighborhood of $x$.

$$
M(s x, s y, t) \geq r, \quad N(s x, s y, t) \leq 1-r
$$

for all $s \in S$ and all $y \in O$. Hence $x \in \operatorname{Eq}(X)$. This completes the proof.

\section{Acknowledgment}

This research was partially supported by the NSF of China (Grant 10771056) and the Special Research Found for the Doctoral Program of Higher Education. 


\section{References}

[1] L. A. Zadeh, "Fuzzy sets," Information and Control, vol. 8, no. 3, pp. 338-353, 1965.

[2] K. T. Atanassov, "Intuitionistic fuzzy sets," Fuzzy Sets and Systems, vol. 20, no. 1, pp. 87-96, 1986.

[3] D. Coker, "An introduction to intuitionistic fuzzy topological spaces," Fuzzy Sets and Systems, vol. 88, no. 1, pp. 81-89, 1997.

[4] S. N. Ješić and N. A. Babačev, "Common fixed point theorems in intuitionistic fuzzy metric spaces and $L$-fuzzy metric spaces with nonlinear contractive condition," Chaos, Solitons \& Fractals, vol. 37, no. 3, pp. 675-687, 2008.

[5] I. Kramosil and J. Michálek, "Fuzzy metrics and statistical metric spaces," Kybernetika, vol. 11, no. 5, pp. 336-344, 1975.

[6] C. Alaca, D. Turkoglu, and C. Yildiz, "Fixed points in intuitionistic fuzzy metric spaces," Chaos, Solitons \& Fractals, vol. 29, no. 5, pp. 1073-1078, 2006.

[7] A. George and P. Veeramani, "On some results in fuzzy metric spaces," Fuzzy Sets and Systems, vol. 64, no. 3, pp. 395-399, 1994.

[8] J. H. Park, "Intuitionistic fuzzy metric spaces," Chaos, Solitons \& Fractals, vol. 22, no. 5, pp. 1039-1046, 2004.

[9] C. Alaca, H. Efe, and C. Yildiz, "On completion of intuitionistic fuzzy metric spaces," Chaos, Solitons \& Fractals. In press.

[10] M. S. El Naschie, "On the verifications of heterotic string theory and $e(1)$ theory," Chaos, Solitons \& Fractals, vol. 11, pp. 397-407, 2000.

[11] M. S. El Naschie, "A review of $E^{\infty}$ theory and the mass spectrum of high energy particle physics," Chaos, Solitons \& Fractals, vol. 19, no. 1, pp. 209-236, 2004.

[12] L. D. G. Sigalotti and A. Mejias, “On El Naschie's conjugate complex time, fractal $E^{\infty}$ space-time and faster-than-light particles," International Journal of Nonlinear Sciences and Numerical Simulation, vol. 7, no. 4, pp. 467-472, 2006.

[13] L. Chang and L. A. Zadeh, "On fuzzy mapping and control," IEEE Transactions on Systems, Man and Cybernetics, vol. 2, no. 1, pp. 30-34, 1972.

[14] M. de Glas, "Theory of fuzzy systems," Fuzzy Sets and Systems, vol. 10, no. 1-3, pp. 65-77, 1983.

[15] P. E. Kloeden, "Fuzzy dynamical systems," Fuzzy Sets and Systems, vol. 7, no. 3, pp. 275-296, 1982.

[16] L. Chen, H. Kou, M.-K. Luo, and W. Zhang, "Discrete dynamical systems in L-topological spaces," Fuzzy Sets and Systems, vol. 156, no. 1, pp. 25-42, 2005.

[17] G. Cairns, G. Davis, D. Elton, A. Kolganova, and P. Perversi, "Chaotic group actions," Enseignement Mathematique, vol. 41, pp. 123-133, 1995.

[18] G. Cairns and T. D. Pham, "An example of a chaotic group action on Euclidean space by compactly supported homeomorphisms," Topology and Its Applications, vol. 155, no. 3, pp. 161-164, 2007.

[19] E. Kontorovich and M. Megrelishvili, "A note on sensitivity of semigroup actions," Semigroup Forum, vol. 76, no. 1, pp. 133141, 2008.

[20] E. Glasner and B. Weiss, "Sensitive dependence on initial conditions," Nonlinearity, vol. 6, no. 6, pp. 1067-1075, 1993. 

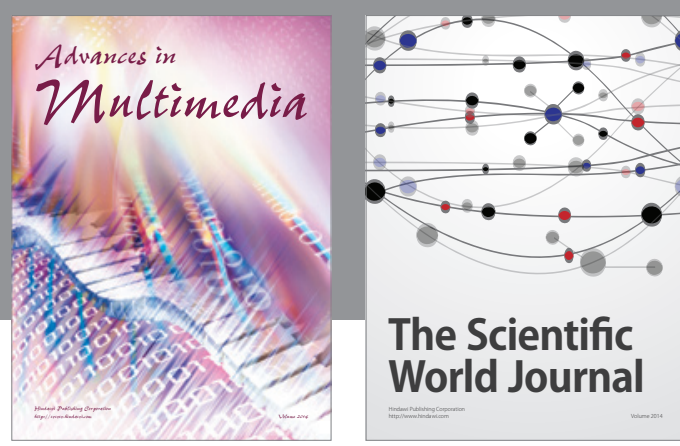

The Scientific World Journal
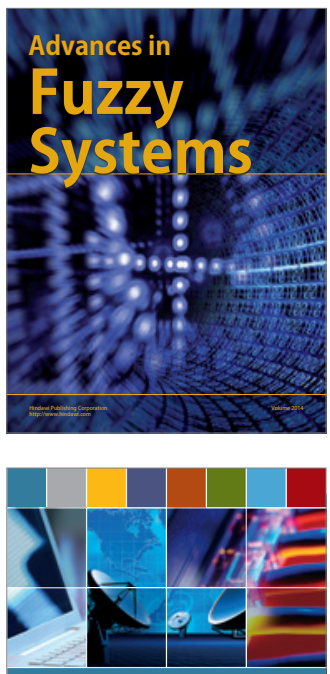

Computer Networks and Communications
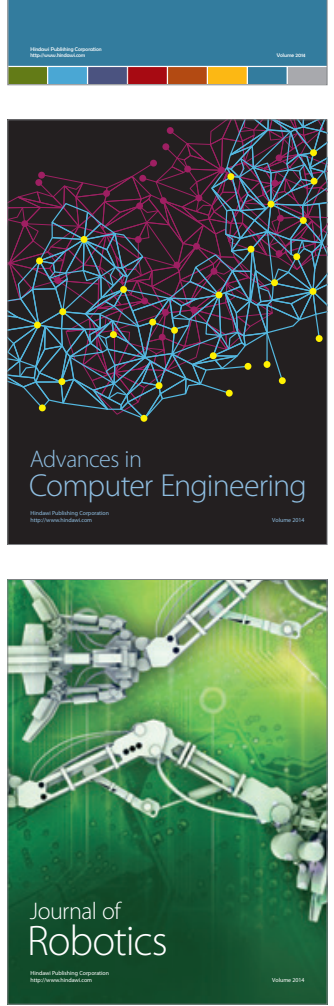
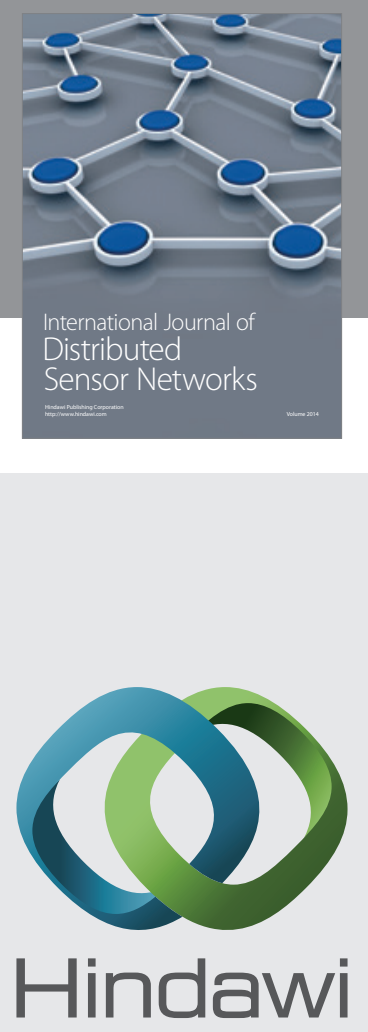

Submit your manuscripts at

http://www.hindawi.com
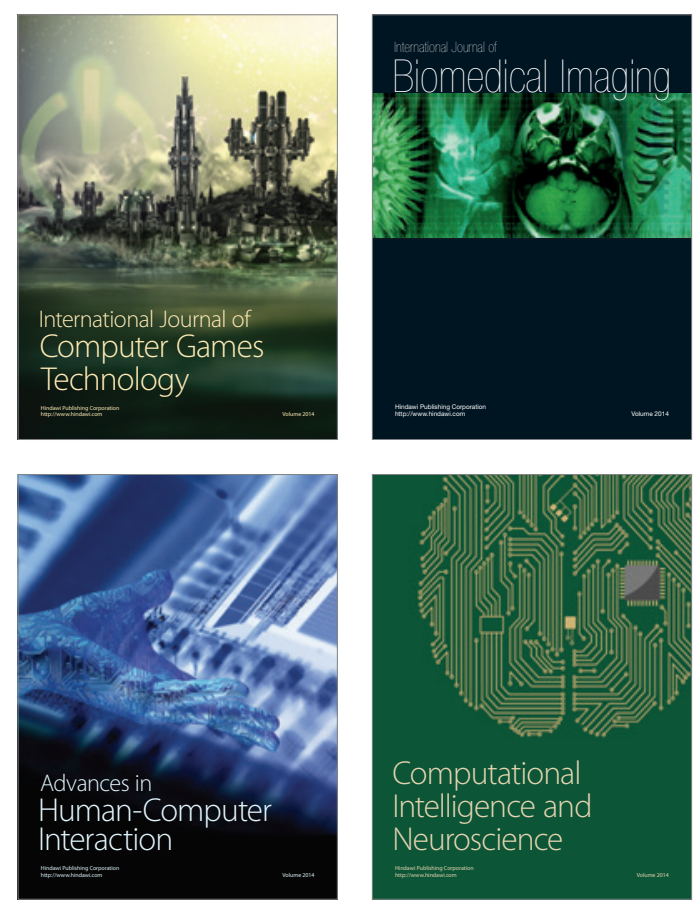
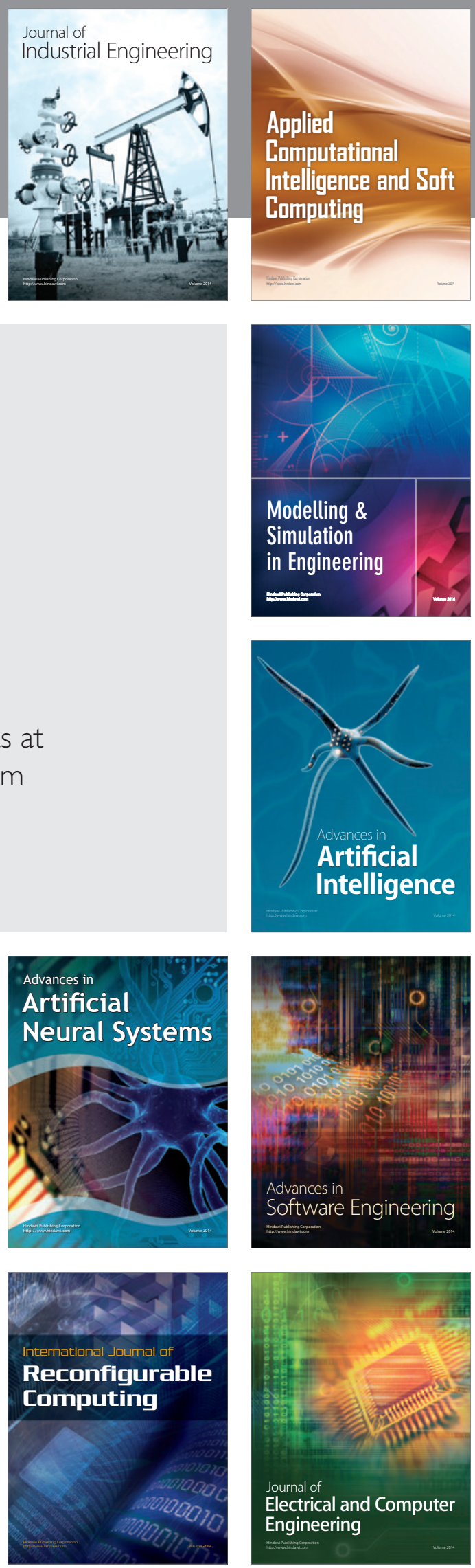